\title{
Carbon Stars in the Magellanic Clouds
}

\author{
M. Azzopardi \\ IGRAP, Observatoire de Marseille, 2 Place Le Verrier, \\ F-13248 Marseille Cedex 4, France
}

\begin{abstract}
Thanks to their pronounced and well recognizable spectral features, carbon stars can be identified easily in Galactic halo and galaxies. In this paper we will review the extensive searches for carbon stars in the Magellanic Clouds with emphasis on the recent work devoted to the carbon stars of low luminosity and the more classical carbon stars populating the outer regions of these systems.
\end{abstract}

\section{Introduction}

The red giant stars are of special interest for the study of the morphology, stellar evolution and kinematics of the galaxies. In addition, the ratio of carbon (C) stars to the late-type $\mathrm{M}$ stars, and possibly the faint-end of the C star luminosity function, give information on the metallicity of the parent system. The envelopes and the atmospheres of Asymptotic Giant Branch (AGB) stars are enriched with molecules formed out of either oxygen or carbon, which make their spectroscopic and/or photometric detection possible in external galaxies as far away as the M81/NGC 2403 group. The excess carbon with regard to oxygen $(\mathrm{C} / \mathrm{O}>1)$ in $\mathrm{C}$ stars allows their identification mainly through their strong $\mathrm{CN}$ and Swan $\mathrm{C}_{2}$ bands. These readily recognized features, combined with the proximity of the Magellanic Clouds, have contributed significantly to the success of the Magellanic Cloud C star surveys for the past four decades. Note that the large extent in the sky of these galaxies and the general relative intrinsic brightness of the classical C stars make the wide field photographic plate surveys still competitive, even in the era of CCD detectors, so long as CCD mosaic cameras are not yet in current use.

\section{Early Carbon Star Surveys}

The discovery of nine C star candidates in the Large Magellanic Cloud (LMC) was first reported by Westerlund (1960). It was the result of a photometric $(R, I)$ and near-infrared objective-prism surveys $(\approx 2100 \AA / \mathrm{mm}$ dispersion at the A-band) of four central regions in the LMC, carried out by the author in the late 1950 s using the $50 / 65 / 173 \mathrm{~cm}$ Schmidt telescope at Uppsala Southern Station, Mt Stromlo Observatory. An extension of this survey $(I>13)$ to the major part of the LMC (62.5 sq. deg.) led to the identification of $302 \mathrm{C}$ star candidates (Westerlund 1964; Westerlund et al. 1978). Meanwhile, the CTIO 
blue-green Curtis-Schmidt telescope objective-prism search $(1360 \AA / \mathrm{mm}$ dispersion at $\mathrm{H} \gamma$ ) by Sanduleak \& Philip (1977) of part of the area previously surveyed by Westerlund led to the detection of 474 LMC C star candidates of which 399 were newly discovered objects. This result shows clearly, that although the nearinfrared low dispersion objective-prism technique favours detection of the late $\mathrm{N}$-type $\mathrm{C}$ stars displaying strong blends of CN-bands near $\lambda \lambda 7910,8100$ and $8320 \AA$, the strength of the Swan $C_{2}$ bands at $\lambda \lambda 4737,5165$ and $5635 \AA$ more than compensates the loss of flux in the blue-green spectral domain of $\mathrm{C}$ stars, hence does not introduce this bias while allowing detection of the hotter ones. Subsequent detailed spectroscopic and photometric studies by Crabtree et al. (1976) and Richer et al. (1979) of selected candidates drawn from those lists were to confirm the carbon star nature and provide information on the physical properties of the first LMC C stars.

Deeper $(I>17)$ near-infrared grism searches $(2350 \AA / \mathrm{mm}$ and $1700 \AA / \mathrm{mm}$ dispersions at the $\mathrm{A}$-band) for $\mathrm{C}$ and late $\mathrm{M}$-type stars in selected fields of the Magellanic Clouds were carried out by Blanco \& McCarthy using a photographic camera equipped with a Schott RG630 filter at the prime focus of the CTIO 4.0- $\mathrm{m}$ reflector: $1045 \mathrm{C}$ star candidates in the LMC and 860 ones in the Small Magellanic Cloud $(\mathrm{SMC})$ were detected in $\approx 6.2$ and $\approx 6.8 \mathrm{sq}$. deg. total fields, respectively (Blanco et al. 1980; Blanco \& McCarthy 1983, 1990). Carbon starcount isopleths based on the surface densities determined for these objects in 52 LMC sample areas and 37 SMC ones led Blanco \& McCarthy (1983) to estimate the total numbers of cool C stars as 11000 in the LMC and 2900 in the SMC. Also, study of the distributions of $\mathrm{M}$ giants led these authors to investigate the ratios of the frequencies of $\mathrm{C}$ and $\mathrm{M}$-type stars in the Magellanic Clouds. They found that the $\mathrm{C} / \mathrm{M}$ ratio, i.e. (number of $\mathrm{C}$ stars)/(number of giants of type M6 or later) is $2.2 \pm 0.1$ in the LMC and appears to be constant throughout the galaxy; for the SMC, this ratio is $19.2 \pm 0.8$ in the central regions and decreases continuously outwards to reach $4.7 \pm 0.4$ in its periphery. If $\mathrm{C} / \mathrm{M}$ is related to metallicity alone - not also a function of the red giant age - this lets us ascertain that no significant metallicity gradient exists in the LMC, while for the SMC an increase of metallicity toward the external regions is indicated at the time, 3-5 Gyr ago, when the red giants were formed.

An extensive photographic slitless spectroscopy survey for field C stars in the SMC was achieved by us (see Azzopardi \& Westerlund 1984) at the prime focus of the ESO $3.6-\mathrm{m}$ telescope. We used the wide field corrector equipped with a blue-green grism ( $2200 \AA / \mathrm{mm}$ dispersion) in combination with a Schott GG 435 filter and IIIa-J plates in order to restrict the instrumental spectral range to a useful domain (4300-5300 $\AA$ ) and keep the number of spectrum overlaps as low as possible. The main body of the SMC - except its western regions - was covered by 13 partially overlapping fields, $0.78 \mathrm{deg}^{2}$ each. Adopting the $\mathrm{C}$ star detection technique innovated by Sanduleak \& Philip (1977) (search for Swan $\mathrm{C}_{2}$ band features) we found $1707 \mathrm{C}$ star candidates (Rebeirot et al. 1993). Our $\mathrm{C}$ star detection is basically complementary to the near-infrared one (search for $\mathrm{CN}$ bands) by Blanco and associates (see McCarthy 1987). The overall surface distribution of the SMC C stars shows that they seem to belong to a spheroidallike system, in fairly good agreement with the smoothed isophotes of the SMC in red light (see de Vaucouleurs \& Freeman 1972). 


\section{Low-Luminosity Carbon Stars in the Magellanic Clouds}

The relation between the carbon abundance and a colour-equivalent given by the spectrophotometry of almost all C stars provided by the catalogue of Rebeirot et al. (1993) allowed us to detect natural groups and especially, to identify a sample $(\approx 5 \%$ of the set) of faint and relatively blue objects. Subsequent ESO medium resolution spectroscopy and $J H K$ photometry of 50 of them led Westerlund et al. $(1992,1995)$ to discover SMC C stars at the highest temperature and lowest luminosity ever found in an external galaxy. Up to now the faint-end of the SMC C star luminosity function inferred from 161 stars is $M_{\text {bol }} \approx-1.4 \mathrm{mag}$, assuming $(m-M)_{0}=18.8$ as the absorption-free distance modulus for the parent system (see Azzopardi 1994).

Using the same selection criteria, we are now looking for faint $\mathrm{C}$ stars in the LMC from the spectrophotometry of about 1200 objects identified in eight selected fields which have been obtained - as a backup programme - during our observations of the SMC. Work is in progress, but so far we have not found any $\mathrm{C}$ star fainter than $M_{\mathrm{bol}}=-3.3 \mathrm{mag}$. This preliminary result as well as our recent discovery of low-luminosity $\mathrm{C}$ stars in the Fornax dwarf spheroidal galaxy - resembling those we had previously identified in the SMC, another sligthly metal-poor galaxy - corroborates the theoretical predictions leading to the expectation that the mean luminosity of $\mathrm{C}$ stars should be lower in the more metal-deficient system. According to Frantsman (1997, and these proceedings) the low-luminosity C stars may be in the early AGB evolutionary stage and should be "formed as a result of the mass transfer in close binary systems while the primary is a C star on the thermally-pulsing AGB stage" (see also in these proceedings the paper by Marigo \& Girardi and references quoted therein).

\section{Carbon Stars in the Magellanic Cloud Haloes}

Investigations of the dynamical interactions between the components of the Galaxy-LMC-SMC system for a better understanding of the morphology of the SMC and the LMC as well as the attempts to determine the total mass of these galaxies has incited searches for suitable kinematic tracers in the outer regions of the Magellanic Clouds. Jointly, the discovery of $\mathrm{CH}$ stars in the outer halo of the LMC by Hartwick \& Cowley (1988), strengthened by their intrinsic brightness (see Feast \& Whitelock 1992), has clearly emphasized the usefulness of the carbon-type stars for this kind of study. In consequence extensive surveys for $\mathrm{C}$ stars in the outer haloes of the Magellanic Clouds, including the inter-Cloud region, and subsequent spectroscopic observations for kinematic purposes have come out recently.

The UK Schmidt Telescope (UKST) objective-prism ( $800 \AA / \mathrm{mm}$ dispersion near $4300 \AA$ ) survey of $\mathrm{C}$ stars in the outer parts of the SMC by Morgan \& Hatzidimitriou (1995), covering a total area of $\approx 220$ sq. deg. on the sky, resulted in the detection of 1185 additional candidates. Coupling this survey with that of the inner regions of the SMC by Rebeirot et al. (1993), Morgan \& Hatzidimitriou estimated that the total number of $\mathrm{C}$ stars in the $\mathrm{SMC}$ is $\simeq 3060$. Radial velocities of a sample of $71 \mathrm{C}$ stars selected in the outer parts of the SMC allowed Hatzidimitriou et al. (1997) to determine the mean heliocentric velocity 
of these stars to be $149.3 \pm 3.0 \mathrm{~km} / \mathrm{s}$ with a velocity dispersion of $25.2 \pm 2.1 \mathrm{~km} / \mathrm{s}$ which lead to a SMC mass of $\simeq 1.210^{9} M_{\odot}$. Similar searches by Demers et al. (1993) and Kunkel et al. (1997a) in the inter-Cloud region and in the Magellanic Cloud haloes from the photometry of UKST $B_{J}$ and $R$ plates (19 fields) resulted in the identification of over one thousand $C$ stars. Radial velocities of $759 \mathrm{C}$ stars on the periphery of the LMC led Kunkel et al. (1997b) to determine the rotation curve from 3 to $12 \mathrm{kpc}$ and the mass contained within a $5 \mathrm{kpc}$ radius, $6.2 \pm 0.9$ $10^{9} M_{\odot}$ (see also the papers by Demers and Kunkel in these proceedings).

\section{References}

Azzopardi, M. 1994, in The Local Group: Comparative and Global properties, A. Layden, R.C. Smith \& J. Storm, ESO Conference and Workshop Proceedings No. 51,129

Azzopardi, M., \& Westerlund, B.E. 1984, The Messenger, 36, 12

Blanco, V.M., \& McCarthy, M.F. 1983, AJ, 88, 1442

Blanco, V.M., \& McCarthy, M.F. 1990, AJ, 100, 674

Blanco, V.M., McCarthy, M.F., \& Blanco, B.M. 1980, ApJ, 252, 133

Crabtree, D.R., Richer, H.B., \& Westerlund, B.E. 1976, ApJ, 203, L81

Demers, S., Irwin, M.J., \& Kunkel, W.E. 1993, MNRAS, 260, 103

de Vaucouleurs, G., \& Freeman, K.C. 1972, Vistas in Astronomy, 14163

Feast, M.W., \& Whitelock, P.A. 1992, MNRAS, 259, 6

Frantzman, J. 1997, A\&A, 319, 511

Hartwick, F.D.A., \& Cowley, A.P. 1988, ApJ, 334, 135

Hatzidimitriou, D., Croke, B.F., Morgan, D.H., \& Cannon, R.D 1997, A\&AS, 122,507

Kunkel, W.E., Irwin, M.J., \& Demers, S. 1997a, A\&AS, 122, 463

Kunkel, W.E., Demers, S., Irwin, M.J., \& Albert, L. 1997b, ApJ, 488, L129

McCarthy, M.F. 1987, in Stellar Evolution and Dynamics of the Outer Halo of the Galaxy, M. Azzopardi \& F. Matteucci, ESO Conference and Workshop Proceedings No.27, 203

Morgan D.H., \& Hatzidimitriou, D. 1995, A\&AS, 113, 539

Rebeirot, E., Azzopardi, M., \& Westerlund, B.E. 1993, A\&AS, 97603

Richer, H.B., Olander, N., \& Westerlund, B.E. 1979, ApJ, 230, 724

Sanduleak, N., \& Philip, A.G.D. 1977, Publ. Warner Swasey Obs. 2, 105

Westerlund, B.E. 1960, Uppsala Astron. Obs. Ann. 4, No. 7

Westerlund, B.E. 1964, in IAU/URSI Symp. No. 20, F.J. Kerr \& A.W. Roggers, Australian Academy of Science, Canberra, Australia, 239

Westerlund, B.E., Olander, N., Richer, H.B., \& Crabtree, D.R. 1978, A\&AS, 31,61

Westerlund, B.E., Azzopardi, M., Breysacher, J., \& Rebeirot, E. 1992, A\&A, 260, L4

Westerlund, B.E., Azzopardi, M., Breysacher, J., \& Rebeirot, E. 1995, A\&A, 303,107 